\title{
Violating Suydam criterion produces feeble instabilities
}

\author{
Sangeeta Gupta, J. D. Callen, and C. C. Hegna \\ Department of Engineering Physics, University of Wisconsin, Madison, Wisconsin 53706-1687
}

(Received 13 February 2002; accepted 28 April 2002)

\begin{abstract}
Eigenmode analysis of a magnetic shear localized ideal interchange mode indicates a marginal stability condition consistent with the Suydam (and Mercier) criterion. However, the linear eigenmode analysis shows that it is not a very restrictive condition because the mode growth rate is exponentially small just above the marginal stability condition and the modes are easily stabilized by finite ion Larmor radius (FLR) effects. This suggests that for practical applications to toroidal confinement experiments, the Suydam-Mercier criterion does not introduce a restrictive condition for operational limits. The criterion for robust growth appears to be almost a factor of two greater than the Suydam criterion. It is also shown that even the presence of usual nonideal effects (FLR, electron diamagnetic flow and resistivity) does not alter this higher criterion for robust growth. (C) 2002 American Institute of Physics. [DOI: 10.1063/1.1487865]
\end{abstract}

\section{INTRODUCTION}

Magnetohydrodynamics (MHD) is quite successful in determining macroscopic equilibrium properties and linear stability boundaries of a toroidal magnetically confined plasma. The theoretical stability boundaries are defined using an energy principle approach for which stability is analyzed by examining the sign of $\delta W$, where $\delta W$ is the change in potential energy caused by a small perturbation. The system is stable if $\delta W \geqslant 0$ for all allowable displacements satisfying the relevant boundary conditions. The stability condition for a localized ideal interchange mode in a cylindrical plasma is known as Suydam's criterion, ${ }^{1,2}$ which is

$$
D_{I} \leqslant 1 / 4, \quad \text { for stability. }
$$

Here, the driving term for instability is $D_{I} \equiv-\left(8 \pi p_{0}^{\prime} /\right.$ $\left.B_{z}^{2} r_{s}\right)\left(q / q^{\prime}\right)^{2} \equiv \beta L_{s}^{2} / r_{p} R_{c}$ in which $1 / L_{s} \equiv\left(1 / R_{0} q\right)\left(r q^{\prime} / q\right)$, $\beta / r_{p} \equiv-8 \pi P_{0}^{\prime} / B_{z}^{2}$ and $R_{c}$ is the average magnetic-field curvature radius. In a toroidal geometry, the stability regime is given by the Mercier criterion, ${ }^{1,3}$ again with $D_{I} \leqslant 1 / 4$ for stability. For an axisymmetric tokamak in the limit of large aspect ratio, small $\beta$ and circular cross-section, the form of $D_{I}$ in the Mercier criterion is $D_{I}=-\left(8 \pi p_{0}^{\prime} / B_{\phi}^{2} r\right)$ $\times\left(q / q^{\prime}\right)^{2}\left(1-q^{2}\right){ }^{1,4}$ In these derivations, the mode is assumed to be localized arbitrarily close to the mode rational surface.

The above criteria, derived using the energy principle, do not provide any information about the properties of the unstable mode once the threshold is exceeded. Earlier, attempts have been made to solve the linear eigenmode equation for the localized ideal interchange mode in the context of stellarators and pinches using cylindrical models. ${ }^{5,6}$ Those analysis indicated that the growth rate is exponentially small for $D_{I}>1 / 4$ and that the mode can be easily stabilized by finite ion Larmor radius effects. However, in the presence of plasma electrical resistivity, resistive-g or resistive interchange modes become unstable for $D_{I}>0 .^{7-9}$ The growth rate of the resistive interchange mode scales as $\gamma \propto \eta^{1 / 3} D_{I}^{2 / 3}$ where $\eta$ is the resistivity. In the presence of electron diamag- netic and ion finite Larmor radius effects, the growth rate of resistive interchange mode is significantly reduced. ${ }^{8}$

In this paper, we describe an analysis of linear stability properties of ideal and resistive interchange instabilities using a simple sheared slab model. Here, all the relevant effects such as ion finite Larmor radius effects, plasma resistivity and electron diamagnetic flow effects are included in one coherent "simple" slab model. We show, both analytically and numerically, that the growth rate of a localized ideal interchange mode is exponentially small for $D_{I}$ well above the marginal stability condition and is easily stabilized by the ion finite Larmor radius (FLR) effects. The effective stability criterion appears to be $D_{I} \leq 0.4-0.5$ rather than the Suydam criterion $D_{I}<1 / 4$. A physical interpretation of these results is given in terms of the energy principle.

In the presence of dissipative effects such as resistivity, the eigenmode structure changes significantly. The reason is that the singular layer is resolved by the resistive layer rather than by the inertial layer as in ideal MHD case. Since resistivity changes the eigenmode structure, an energy principle analysis is no longer valid. Our eigenmode analysis for the resistive interchange instability indicates that the mode grows on a resistive time scale similar to that of equilibrium evolution, in the presence of electron diamagnetic and finite Larmor radius effects. Thus, it seems that even in the presence of nonideal effects such as resistivity, electron diamagnetic flow and ion finite Larmor radius, the criterion for a robust (ideal MHD-like) growth is greater than the Suydam's criterion by almost a factor of 2 .

This paper is organized as follows. Section II describes the basic model used for studying the localized ideal and resistive interchange instabilities. In Sec. III, the eigenvalue equation for the ideal interchange mode is solved both analytically and numerically. The resistive interchange instability is discussed in Sec. IV. Finally, conclusions are presented in Sec. V. 


\section{BASIC MODEL}

We consider a sheared slab model where the magnetic field is locally represented by

$$
\vec{B}=B_{0}\left[\left(1+\frac{x}{L_{B}}\right) \hat{e}_{z}+\frac{z}{R_{c}} \hat{e}_{x}+\frac{x}{L_{s}} \hat{e}_{y}\right] .
$$

Here $\hat{e}_{x}, \hat{e}_{y}$, and $\hat{e}_{z}$ are unit vectors along $x, y$ and $z, L_{B}$ $=(d \ln B / d x)^{-1}$ is the scale-length of the perpendicular gradient of magnetic-field strength, $L_{s}=B\left(d B_{y} / d x\right)^{-1}$ is the magnetic shear scale length and $\kappa=-1 / R_{c}$ is the curvature of the magnetic field. Here, $R_{c}>0$ represents good curvature and $R_{c}<0$ represents bad curvature. In equilibrium, the radial force balance equation gives $d\left(P_{0}+B^{2} / 8 \pi\right) / d x$ $=B_{0} B_{z} / 4 \pi R_{c}$, which implies $\beta / r_{p}=1 / R_{c}-1 / L_{B}$. On the other hand, in the direction parallel to the equilibrium magnetic field, pressure is constant, i.e., $\nabla_{\|} P_{0}=0$.

To derive an eigenvalue equation for ideal and resistive interchange instabilities, we consider a low $\beta$ plasma (i.e., $\beta \ll 1$ ), which allows us to neglect the compressional component of magnetic-field perturbations. Thus, we can write $\vec{E}_{\perp}=-\vec{\nabla}_{\perp} \phi_{1}$ and $E_{\|}=-\nabla_{\|} \phi_{1}-(1 / c) \partial A_{\|} / \partial t$ where $\phi_{1}$ is the scalar potential perturbation and $A_{\|}\left[\equiv\left(\vec{A}_{1} \cdot \vec{B}\right) /|B|\right]$ is the parallel vector potential perturbation. We also assume that the plasma is incompressible (i.e., $\vec{\nabla} \cdot \vec{V}=0$ ), which eliminates sound wave coupling. Then, the linearized MHD equations, in the presence of nonideal effects such as resistivity, electron diamagnetic and ion finite Larmor radius, are

$$
\begin{aligned}
\rho_{m} & \frac{c^{2}}{B^{2}}\left(\frac{d}{d t}+\vec{V}_{* i} \cdot \vec{\nabla}\right) \nabla_{\perp}^{2} \phi_{1} \\
& +\frac{c}{B^{2}} \vec{B} \times \vec{\nabla} p_{1} \cdot(\vec{\nabla} \ln B+\vec{\kappa})+(\vec{B} \cdot \vec{\nabla})\left(\frac{J_{\|}}{B}\right)=0,
\end{aligned}
$$

$\vec{V}_{1}=\frac{c}{B^{2}} \vec{B} \times \vec{\nabla} \phi_{1}$,

$$
\begin{aligned}
& \left(\frac{\partial}{\partial t}+\vec{V}_{* e} \cdot \vec{\nabla}-\frac{\eta c^{2}}{4 \pi} \nabla_{\perp}^{2}\right) \frac{A_{\|}}{c}=-\nabla_{\|} \phi_{1}-\frac{1}{e n} \nabla_{\|} p_{e 1}, \\
& J_{\|}=-\frac{c}{4 \pi} \nabla_{\perp}^{2} A_{\|}, \quad \frac{\partial p_{1}}{\partial t}=-\vec{V}_{1} \cdot \vec{\nabla} P_{0},
\end{aligned}
$$

where $J_{\|} \equiv\left(\vec{B} \cdot \vec{J}_{1}\right) /|B|$ is the parallel component of the current perturbation, $\vec{V}_{* e} \equiv-\left(c T_{e} / e B^{2}\right) \vec{B} \times \vec{\nabla} \ln P_{e 0}$ is the electron diamagnetic flow and $\vec{V}_{* i} \equiv\left(c T_{i} / e B^{2}\right) \vec{B} \times \vec{\nabla} \ln P_{i 0}$ is the ion diamagnetic flow. The lowest order ion finite Larmor radius effects are derived by taking into account the gyroviscous part of ion stress tensor and they enter via diamagnetic flow effects in the polarization current. ${ }^{10}$ The electron diamagnetic and resistive effects are included by taking into account electron pressure and resistive terms in the parallel Ohm's law. Here, an adiabatic equation of state is considered for both electrons and ions. The subscript 0 represents equilibrium quantities while the subscript 1 represents linear perturbations.

With perturbations of the form $f(\vec{x}, t)=f(x) \exp \left[i\left(k_{y} y\right.\right.$ $-\omega t)], \nabla_{\|}=i k_{\|}(x)=i k_{y} x / L_{s}$, Eqs. (3) $-(5)$ can be combined into two coupled differential equations

$$
\begin{aligned}
& {\left[\omega\left(\omega-\omega_{* i}\right)\left(\frac{\partial^{2}}{\partial x^{2}}-k_{y}^{2}\right)-\frac{k_{y}^{2} c_{s}^{2}}{R_{c} r_{p}}\right] \phi} \\
& \quad-\omega k_{y} \frac{x}{L_{s}} V_{A}^{2}\left(\frac{\partial^{2}}{\partial x^{2}}-k_{y}^{2}\right) \frac{A_{\|}}{c}=0, \\
& {\left[\omega-\omega_{* e}-i \frac{\eta c^{2}}{4 \pi}\left(\frac{\partial^{2}}{\partial x^{2}}-k_{y}^{2}\right)\right] \frac{A_{\|}}{c}=k_{y} \frac{x}{L_{s}} \frac{\left(\omega-\omega_{* e}\right)}{\omega} \phi,}
\end{aligned}
$$

where $\omega_{* e} \equiv k_{y} a_{s} c_{s} / r_{p}$ is the electron diamagnetic frequency, $\omega_{* i} \equiv-\tau \omega_{* e}$ is the ion diamagnetic frequency, $a_{s}$ $\equiv c_{s} / \Omega_{i}$ is the ion Larmor radius at electron temperature $T_{e}$, $c_{s} \equiv \sqrt{T_{e} / m_{i}}$ is the sound speed, $V_{A} \equiv B / \sqrt{4 \pi \rho_{m}}$ is the Alfvén speed, $\Omega_{i} \equiv e B / m_{i} c$ is the ion gyrofrequency, $r_{p}$ $\equiv|d \ln P / d x|^{-1}$ is the pressure gradient scale-length and $\tau$ $\equiv T_{i} / T_{e}$. Here, we have dropped subscript 1 from the potential perturbations $(\phi)$.

\section{IDEAL INTERCHANGE INSTABILITY}

In the absence of nonideal effects, i.e., resistivity, electron diamagnetic and finite Larmor radius (FLR) effects, the eigenvalue equation for the ideal interchange mode can be obtained by combining Eqs. (6) and (7)

$$
\begin{aligned}
\frac{d}{d x}[ & \left.\left(\omega^{2}-\frac{V_{A}^{2}}{L_{s}^{2}} k_{y}^{2} x^{2}\right) \frac{d \phi}{d x}\right] \\
& -k_{y}^{2}\left[\omega^{2}+\frac{c_{s}^{2}}{R_{c} r_{p_{o}}}-k_{y}^{2} x^{2}\left(\frac{V_{A}^{2}}{L_{s}^{2}}-\frac{c_{s}^{2}}{R_{c} r_{p_{o}}} \frac{1}{k_{y}^{2} L^{2}}\right)\right] \phi=0 .
\end{aligned}
$$

Here, we have assumed $1 / r_{p}(x)=\left(1 / r_{p_{o}}\right)\left(1-x^{2} / L^{2}\right)$. In the small (or no) magnetic shear case, this eigenvalue equation becomes a Weber equation. ${ }^{11}$ In the limit $k_{y} L \gg 1$, the mode is always unstable for a magnetic hill (bad curvature) with a growth rate $\gamma \simeq c_{s} / \sqrt{R_{c} r_{p_{o}}}$. This is a valid model for a magnetic mirror ${ }^{12,13}$ and an octopole with no magnetic shear. ${ }^{14}$

On the other hand, in most toroidal plasmas typically $1 / L_{s}^{2}>\left(\beta / R_{c} r_{p}\right)\left(1 / k_{y} L\right)^{2}$, magnetic shear dominates, $r_{p}(x)$ $\simeq r_{p_{o}}$ and the eigenvalue equation can be written in the normalized form:

$$
\frac{d}{d X}\left[\left(\hat{\omega}^{2}-X^{2}\right) \frac{d \phi}{d X}\right]-\left[\hat{\omega}^{2}-X^{2}+D_{I}\right] \phi=0 .
$$

Here, we have defined a normalized distance $X \equiv k_{y} x$ and frequency $\hat{\omega} \equiv \omega /\left(V_{A} / L_{s}\right), \quad D_{I} \equiv \beta L_{s}^{2} /\left(R_{c} r_{p_{o}}\right) \quad$ and $\beta$ $\equiv 8 \pi P_{0} / B^{2} \equiv c_{s}^{2} / V_{A}^{2}$. The terms with coefficient $\hat{\omega}^{2}$ arise from the divergence of the polarization current, $X^{2}$ represents the magnetic-field line bending terms brought about by magnetic shear and $D_{I}$ is the combination of the pressure gradient and curvature effects which represents the source of free energy. This equation can be derived from the standard screw pinch model, given by Eq. (9.45) of Ref. 1, for an incompressible or low $\beta$ plasma where the coupling to sound waves is negligible.

We now solve the eigenvalue equation, Eq. (9), analytically using a matched asymptotic analysis method. ${ }^{15}$ The matching procedure is valid for $\left|\hat{\omega}^{2}\right| \ll 1$. Since the equation 
is symmetric in $X$, it is sufficient to solve this equation only for $X \geqslant 0$. In the outer region, i.e., $\left|X^{2}\right| \gg\left|\hat{\omega}^{2}\right|$, Eq. (9) becomes

$$
\frac{d}{d X}\left(X^{2} \frac{d \phi}{d X}\right)+\left(D_{I}-X^{2}\right) \phi=0 .
$$

In the limit $D_{I} \gg X^{2}$, this equation is the same as that analyzed by Suydam ${ }^{1,2}$ from which he obtained the stability criterion $D_{I} \leqslant 1 / 4$. With $Z=2 X$ and $\phi=\hat{\phi} / Z$, Eq. (10) becomes a Whittaker equation. ${ }^{11,16}$ The Whittaker function solution for a growing mode, which decays as $X \rightarrow \infty$, is given by

$$
\phi_{o}=A_{o} \frac{\exp (-X)}{\sqrt{2 X}}(2 X)^{i \lambda} U\left(\frac{1}{2}+i \lambda, 1+2 i \lambda, 2 X\right),
$$

where

$$
\lambda=\sqrt{D_{I}-\frac{1}{4}}
$$

and $U$ is Kummer's confluent hypergeometric function.

In the inner region, i.e., $\left|X^{2}\right| \sim\left|\hat{\omega}^{2}\right|$, with $Z=X^{2} / \hat{\omega}^{2}$ and $\hat{\omega}^{2} \ll 1$, Eq. (9) reduces to

$$
Z(1-Z) \frac{d^{2} \phi}{d Z^{2}}+\left(\frac{1}{2}-\frac{3}{2} Z\right) \frac{d \phi}{d Z}-\frac{D_{I}}{4} \phi=0,
$$

which is a hypergeometric equation ${ }^{16}$ whose general solution is

$$
\phi_{i}=A_{i} F\left(a, a^{*} ; \frac{1}{2} ; Z\right)+B_{i} \sqrt{Z} F\left(b, b^{*} ; \frac{3}{2} ; Z\right) .
$$

Here $a=1 / 4+i \lambda / 2, b=3 / 4+i \lambda / 2$ and $a^{*}$ and $b^{*}$ represent complex conjugate of $a$ and $b$, respectively. The choice of the coefficients $A_{i}, B_{i}$ depends on the parity condition at $X$ $=0$. For even modes, the boundary condition at $X=0$ is $d \phi / d X=0$, which demands that $B_{i}=0$. For odd modes, the boundary condition at $X=0$ is $\phi=0$, which ensures that $A_{i}$ $=0$. Here, we consider only the even mode solution because it is the most unstable mode.

Next, we match the inner $\phi_{i}$ and the outer $\phi_{o}$ solutions in their overlap region $(|X| \sim|\hat{\omega}|)$. The inner limit of the outer solution [Eq. (11) with $X \rightarrow 0$ ] is

$$
\phi_{o}^{i} \sim A_{o} \sqrt{\frac{\pi}{2 X}}\left(\frac{1}{2 \lambda \Gamma_{+} \sinh \lambda \pi}\right)\left[1+\left(\frac{2}{X}\right)^{2 i \lambda} \frac{\Gamma_{+}}{\Gamma_{-}}\right]\left(\frac{X}{2}\right)^{i \lambda},
$$

and the outer limit of the inner solution [Eq. (14) as $X \rightarrow \infty$ ] is

$\phi_{i}^{o} \sim A_{i} \frac{\sqrt{\pi} \Gamma_{+}}{[\Gamma(a)]^{2}}\left(\frac{X^{2}}{-\hat{\omega}^{2}}\right)^{-a^{*}}\left[1+\frac{\Gamma_{-}}{\Gamma_{+}}\left(\frac{\Gamma(a)}{\Gamma\left(a^{*}\right)}\right)^{2}\left(\frac{-\hat{\omega}^{2}}{X^{2}}\right)^{i \lambda}\right]$,

where $\Gamma_{ \pm}=\Gamma( \pm i \lambda)$. Matching the inner limit of the outer solution to the outer limit of the inner solution results in the eigenvalue equation

$$
\left(\frac{-\hat{\omega}^{2}}{4}\right)^{i \lambda / 2}=-\frac{\Gamma(1+i \lambda)}{\Gamma(1-i \lambda)} \frac{\Gamma(1 / 4-i \lambda / 2)}{\Gamma(1 / 4+i \lambda / 2)} .
$$

This gives a purely growing mode with normalized growth rate $\left(\hat{\gamma}_{\mathrm{MHD}}^{2} \equiv-\hat{\omega}^{2}\right)$
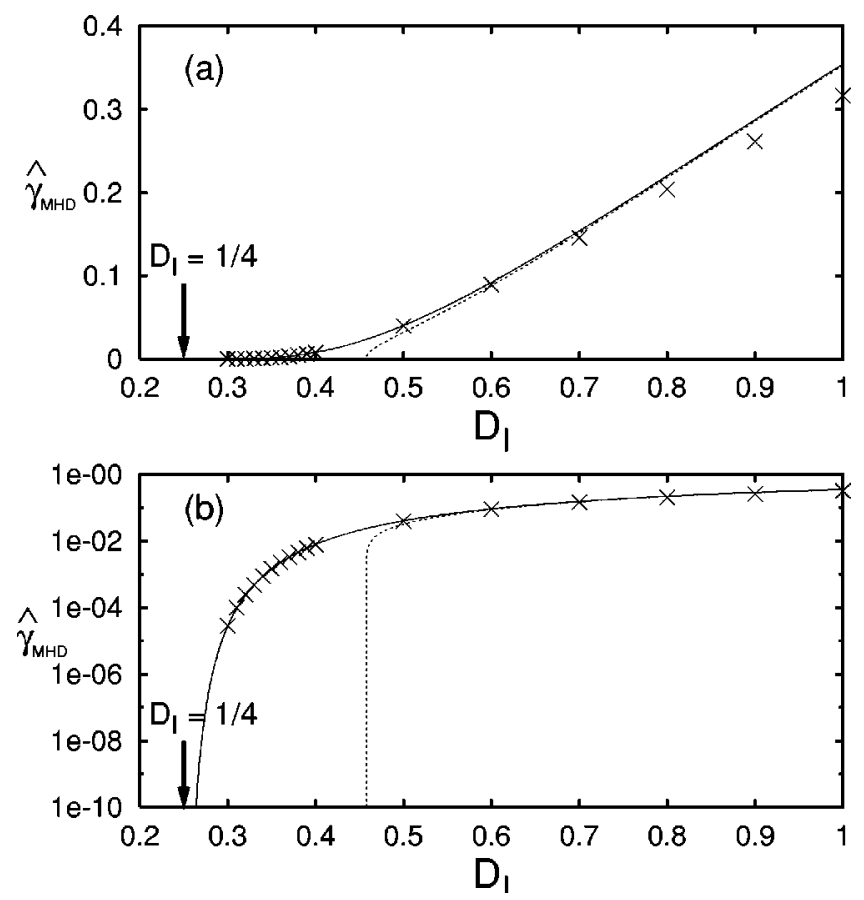

FIG. 1. (a) Normalized growth rate $\hat{\gamma}_{\mathrm{MHD}}$ versus $D_{I}$ shows the agreement between the analytical result (shown by solid line) and the numerical result (represented by $\times$ ). (b) Normalized growth rate on a log scale shows that $\hat{\gamma}_{\text {MHD }}$ is exponentially small well above the marginal stability condition $D_{I}=1 / 4$ (marked by arrow). Ion finite Larmor radius effects stabilize the ideal interchange mode up to $D_{I} \sim 0.46$ for $k_{y} a_{i}=0.04$ and $R_{c} / \tau r_{p}=3$ (shown by the dotted line).

$$
\hat{\gamma}_{\mathrm{MHD}}=\frac{\gamma_{\mathrm{MHD}}}{V_{A} / L_{s}}=2 \exp \left[-\frac{(\pi-2 \Theta)}{|\lambda|}\right],
$$

where $\Theta(|\lambda|) \equiv \arg [\Gamma(i|\lambda|)]+\arg [\Gamma(1 / 4-i|\lambda| / 2)]$ is a function of $|\lambda|$ that asymptotes to zero as $|\lambda| \rightarrow 0$. The above expression indicates clearly that as marginal stability is approached from the unstable region, $D_{I} \rightarrow 1 / 4(|\lambda| \rightarrow 0)$, the normalized growth rate $\hat{\gamma}_{\mathrm{MHD}} \approx \exp (-\pi /|\lambda|) \rightarrow 0$. Thus, the expression for the normalized growth rate gives a marginal stability condition consistent with the Suydam criterion. ${ }^{1,2}$ Similar results have also been observed in Refs. 5 and 6 in more complicated magnetic-field geometries.

Additionally, we have solved the full eigenvalue equation, Eq. (9), numerically using a shooting method with boundary conditions $\phi=0$ at $X=\infty$ and $d \phi / d X=0$ at $X$ $=0$. The analytical results agree very well with numerical results for $\hat{\gamma}_{\mathrm{MHD}}^{2} \ll 1$, as shown in Fig. 1. Figure 1 also shows that the growth rate is still negligible $\left(\hat{\gamma}_{\mathrm{MHD}}<0.03\right)$ even when $D_{I}$ is almost twice the marginal stability condition value (i.e., $D_{I}=1 / 4$ ). For example, for $D_{I}=0.4$, the normalized growth rate $\hat{\gamma}_{\mathrm{MHD}}=0.008$. For large values of $D_{I}$, the normalized growth rate $\hat{\gamma}_{\mathrm{MHD}}$ varies linearly with $D_{I}$ and is approximately given as $\hat{\gamma}_{\mathrm{MHD}} \simeq 0.6\left(D_{I}-0.46\right)$ for $D_{I}>0.5$. The corresponding eigenfunctions for different values of $D_{I}$ are shown in Fig. 2 which indicates that the mode is highly localized around $X=0$. The mode width is a few times the normalized growth rate $\hat{\gamma}_{\mathrm{MHD}}$ and it increases with increasing $D_{I}$ due to the strong increase of $\hat{\gamma}_{\mathrm{MHD}}$ with $D_{I}$ (cf. Fig. 1). 


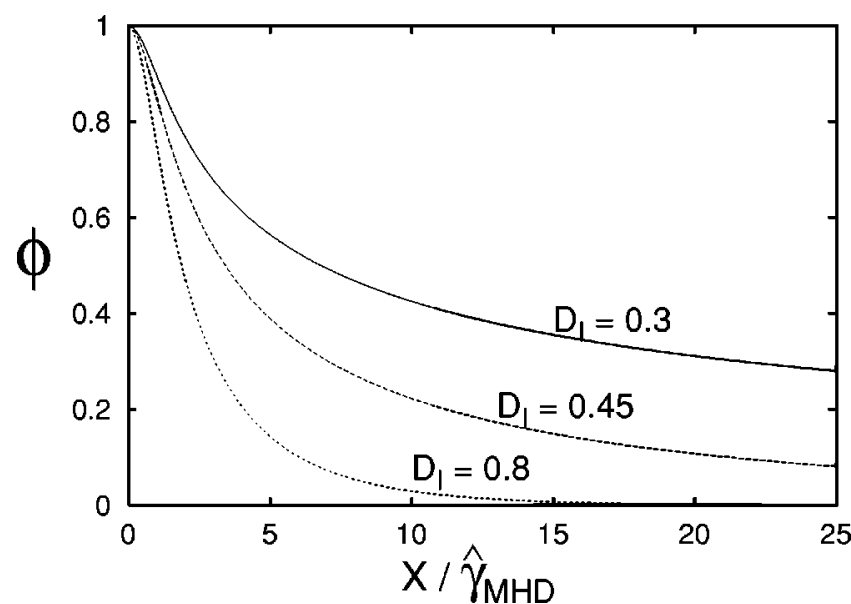

FIG. 2. The eigenfunction versus $X / \hat{\gamma}_{\mathrm{MHD}}$ shows the localization of the mode near $X=0$. Note that its width $\left(\sim \hat{\gamma}_{\mathrm{MHD}}\right)$ in $X$ increases with increasing $D_{I}$ due to increasing $\hat{\gamma}_{\mathrm{MHD}}$ (cf. Fig. 1).

Since the mode width is very narrow for small $D_{I}$, finite ion Larmor radius (FLR) effects become important. Their effect is included by taking into account the gyro-viscous part of ion stress tensor. In the limit $k a_{i}<1$, the ion finite Larmor radius (FLR) effects enter as a diamagnetic flow effect in the polarization term and the eigenmode equation becomes

$$
\begin{aligned}
\frac{d}{d X}[ & {\left[\left(\hat{\omega}\left(\hat{\omega}-\hat{\omega}_{* i}\right)-X^{2}\right) \frac{d \phi}{d X}\right] } \\
& -\left[\hat{\omega}\left(\hat{\omega}-\hat{\omega}_{* i}\right)-X^{2}+D_{I}\right] \phi=0 .
\end{aligned}
$$

Here, $\hat{\omega}_{* i} \equiv \omega_{* i} /\left(V_{A} / L_{s}\right)$ is the normalized ion diamagnetic frequency. In limit $\left|\hat{\omega}\left(\hat{\omega}-\hat{\omega}_{* i}\right)\right| \ll 1$, the dispersion relation with ion FLR effects becomes

$$
\begin{aligned}
& \hat{\omega}^{2}-\hat{\omega} \hat{\omega}_{* i}+\hat{\gamma}_{\mathrm{MHD}}^{2}=0, \\
& \text { or } \hat{\omega}=\frac{\hat{\omega}_{* i}}{2} \pm \sqrt{\frac{\hat{\omega}_{* i}^{2}}{4}-\hat{\gamma}_{\mathrm{MHD}}^{2}},
\end{aligned}
$$

which indicates stability for

$$
\hat{\omega}_{* i}^{2}>4 \hat{\gamma}_{\mathrm{MHD}}^{2} \quad \text { or } \quad k_{y} a_{i}>\frac{2 r_{p}}{L_{s}} \sqrt{\frac{\tau}{\beta}} \hat{\gamma}_{\mathrm{MHD}}=\frac{2 \gamma_{\mathrm{MHD}}}{c_{i} / r_{p}},
$$

where $\hat{\gamma}_{\mathrm{MHD}}\left[\equiv \gamma_{\mathrm{MHD}} /\left(V_{A} / L_{s}\right)\right]$ is given by Eq. (18) and $c_{i}$ $\equiv \sqrt{T_{i} / m_{i}}$ is the ion thermal speed. An example of FLR stabilization effects on the ideal interchange mode is shown in Fig. 1 by the dashed line. Here, FLR effects stabilize the ideal interchange mode for $D_{I} \sim 0.4-0.5$ while for large $D_{I}$ they have negligible effect. Near marginal stability, modes oscillate at the ion diamagnetic frequency, as indicated by Eq. (20).

The eigenmode function remains the same as that in the case of the ideal interchange mode and its mode width is a few times that of the normalized growth rate $\hat{\gamma}_{\mathrm{MHD}}$ for ideal interchange instability. Thus, we can write

$$
\Delta X=k_{y} \Delta x=C \hat{\gamma}_{\mathrm{MHD}},
$$

where $C$ is always greater than 1 (cf. Fig. 2). Thus, the condition $k_{x} a_{i} \sim a_{i} / \Delta x \ll 1$, in the above model for including

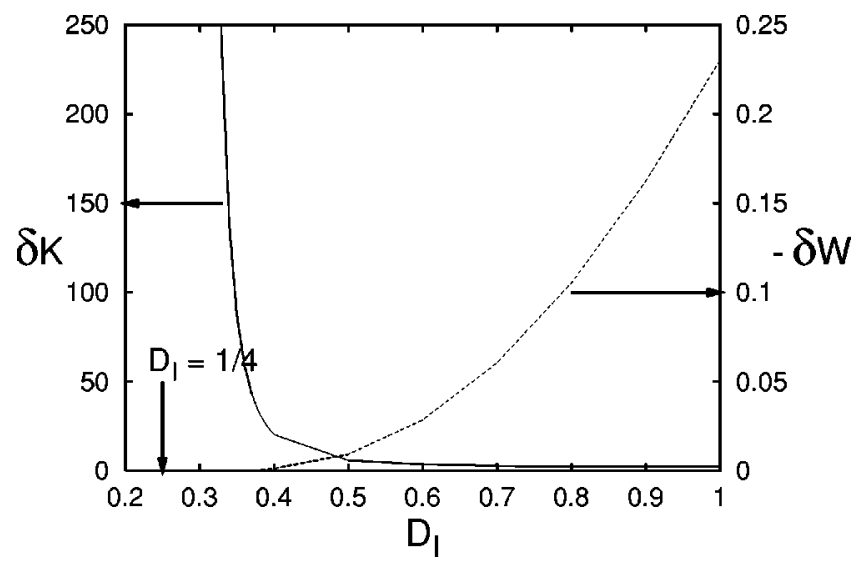

FIG. 3. The potential energy change $-\delta W$ (shown by dashed line) required to drive the instability is negligible even when $D_{I}$ is well above the marginal stability criterion $D_{I}=1 / 4$ (marked by arrow) while the change in kinetic energy $\delta K$ (shown by solid line) is very large for small $D_{I}$.

the finite ion Larmor radius as only the diamagnetic flow effect in the polarization term, can be easily validated for the unstable case if $k_{y} a_{i}<C \hat{\gamma}_{\mathrm{MHD}}$. For example, with $D_{I}=0.5$, the interchange mode is unstable even in the presence of finite ion Larmor radius effects with ideal MHD growth rate $\hat{\gamma}_{\mathrm{MHD}} \sim 0.04$ and $C \sim 5$. This means that $k_{y} a_{i}<0.2$ is required for the validity of our model at this marginal point, which is clearly satisfied in our case (cf. Fig. 1).

\section{Relation to the energy principle}

In order to relate the above results to those with the usual energy principle approach, we now obtain a quadratic form for an ideal interchange mode by multiplying Eq. (9) by $\phi$ and integrating over $X$. For a purely growing mode, i.e., $\hat{\omega} \equiv i \hat{\gamma}_{\mathrm{MHD}}$, we obtain

$$
\begin{aligned}
\hat{\gamma}_{\mathrm{MHD}}^{2} & =\frac{-\delta W}{\delta K} \\
& =\frac{D_{I} \int|\phi|^{2} d X-\int X^{2}\left[|d \phi / d X|^{2}+|\phi|^{2}\right] d X}{\int\left[|d \phi / d X|^{2}+|\phi|^{2}\right] d X} .
\end{aligned}
$$

Here, the numerator is the change in potential energy while the denominator is the change in kinetic energy. The first term in the numerator is the free energy source term due to the combination of pressure gradient and curvature effects while the second term is the stabilizing magnetic field line bending term. Figure 3 shows the variation of $\delta K$ (solid line) and $-\delta W$ (dashed line) with $D_{I}$. It is clear from the figure that for values of $D_{I}$ close to marginal stability, i.e., $D_{I}$ $=1 / 4$, the change in kinetic energy is very large because the mode is highly localized around $X=0$ (cf. Fig. 2), while the potential energy released from the pressure gradient is very small; hence the normalized growth rate $\hat{\gamma}_{\mathrm{MHD}}$ is negligible. For large $D_{I}$, the released potential energy varies nearly quadratically with $D_{I}$ while the change in kinetic energy is nearly constant; this results in a linear variation of $\hat{\gamma}_{\mathrm{MHD}}$ with $D_{I}$ and is approximately given as $\hat{\gamma}_{\mathrm{MHD}} \simeq 0.6\left(D_{I}-0.46\right)$ for $D_{I}>0.5$. 


\section{RESISTIVE INTERCHANGE MODE}

In the presence of plasma electrical resistivity, the normalized coupled equations for the scalar potential $(\phi)$ and the vector potential $\left(A_{\|}\right)$are

$\left[\hat{\omega}\left(\hat{\omega}+\tau \hat{\omega}_{*}\right)\left(\frac{\partial^{2}}{\partial X^{2}}-1\right)-D_{I}\right] \phi-\hat{\omega} X \frac{V_{A}}{c}\left(\frac{\partial^{2}}{\partial X^{2}}-1\right) A_{\|}=0$,

$\left[\hat{\omega}-\hat{\omega}_{*}-i \hat{\nu}_{\eta}\left(\frac{\partial^{2}}{\partial X^{2}}-1\right)\right] A_{\|}=X \frac{c}{V_{A}} \frac{\left(\hat{\omega}-\hat{\omega}_{*}\right)}{\hat{\omega}} \phi$.

Here, we have used the same normalization as before, plus $\hat{v}_{\eta} \equiv k_{y}^{2}\left(\eta c^{2} / 4 \pi\right) /\left(V_{A} / L_{S}\right) \equiv k_{y}^{2} a^{2} / S$. The parameter $\hat{\nu}_{\eta}$ is the normalized damping frequency due to plasma resistance, $S \equiv \tau_{\eta} / \tau_{A}$ is the Lundquist number, $\tau_{\eta} \equiv a^{2} 4 \pi /\left(\eta c^{2}\right)$ is the resistive skin time across the plasma and $\tau_{A} \equiv L_{S} / V_{A}$ is the characteristic Alfvén wave time.

\section{A. Weakly resistive case}

In the weak resistive case with $\hat{\nu}_{\eta} \partial^{2} / \partial^{2} X \sim\left|\hat{\omega}-\hat{\omega}_{*}\right|$ $\ll 1$, Eqs. (22) and (23) can be Fourier transformed into the following second-order differential equation:

$$
\begin{aligned}
& \frac{d}{d k}\left[\frac{\left(\hat{\omega}-\hat{\omega}_{*}\right)\left(1+k^{2}\right)}{\hat{\omega}-\hat{\omega}_{*}+i \hat{\nu}_{\eta}\left(1+k^{2}\right)} \frac{d \hat{\phi}}{d k}\right] \\
& \quad+\left(D_{I}+\hat{\omega}\left(\hat{\omega}+\tau \hat{\omega}_{*}\right)\left(1+k^{2}\right)\right) \hat{\phi}=0,
\end{aligned}
$$

where

$$
\hat{\phi}(k)=\int_{-\infty}^{\infty} d X e^{i X k} \phi(x) .
$$

Here, we obtain an analytical solution of the above equation using a matched asymptotic method. This solution is valid only for $D_{I}<1 / 4$. In the region $k<1$ with $\hat{\nu}_{\eta}<\hat{\gamma}$, the solution of the above equation is given by a hypergeometric function. Its asymptotic form for large $k$ is

$$
\hat{\phi} \sim k^{\delta-1 / 2}\left[A+B k^{-2 \delta}\right],
$$

where $\delta \equiv i \lambda[\lambda$ is defined in Eq. (12)] is a real positive number for $D_{I}<1 / 4$. For the outer region with $k \sim(\hat{\omega}$ $\left.-\hat{\omega}_{*}\right) / \hat{\nu}_{\eta}$, Eq. (24) becomes

$$
\frac{d}{d z}\left[\frac{z^{2}}{1+z^{2}} \frac{d \hat{\phi}}{d z}\right]+\left[D_{I}+\frac{\hat{\omega}\left(\hat{\omega}+\tau \hat{\omega}_{*}\right)\left(\hat{\omega}-\hat{\omega}_{*}\right)}{i \hat{\nu}_{\eta}} z^{2}\right] \hat{\phi}=0,
$$

with $z=\sqrt{i \hat{\nu}_{\eta} /\left(\hat{\omega}+\tau \hat{\omega}_{*}\right)} k$. Its solution is

$$
\hat{\phi}=z^{\alpha} \exp \left(\alpha z^{2} / 2\right) ; \quad \alpha=\frac{1}{2}-i \lambda .
$$

For $D_{I}<1 / 4, \alpha$ is a real positive number lying between 0 and $\frac{1}{2}$. Matching the inner and outer solutions, the corresponding dispersion relation becomes

$$
\hat{\omega}\left(\hat{\omega}+\tau \hat{\omega}_{*}\right)\left(\hat{\omega}-\hat{\omega}_{*}\right)+i \hat{\nu}_{\eta} \alpha^{2}=0 .
$$

In the absence of electron diamagnetic and ion finite Larmor radius effects, the growth rate for modes with $D_{I}<1 / 4$ is

$$
\hat{\gamma}=\hat{\nu}_{\eta}^{1 / 3} \alpha^{2 / 3} \propto\left(k_{y} \alpha\right)^{2 / 3} S^{-1 / 3} .
$$

In the presence of significant FLR and diamagnetic effects with $\hat{\gamma}<\hat{\omega}_{*}$, the mode is purely growing with normalized growth rate

$$
\hat{\gamma}=\frac{\hat{\nu}_{\eta} \alpha^{2}}{\tau \hat{\omega}_{*}^{2}} \propto \alpha^{2} S^{-1} D_{I}^{-1} \sim 1 / S .
$$

Note that this growth rate is on the slow time scale of the resistive equilibrium evolution of the plasma and is independent of wave number. ${ }^{7-9}$

\section{B. Highly resistive case}

In the resistivity dominated case, i.e., $\hat{\nu}_{\eta} \Delta_{\perp}^{2} \gg \hat{\omega}-\hat{\omega}_{*}$, Eqs. (22) and (23) can be combined into a Weber equation

$$
\begin{aligned}
\frac{\partial^{2} \phi}{\partial Z^{2}}+ & {\left[-\left(1+\frac{D_{I}}{\hat{\omega}\left(\hat{\omega}+\tau \hat{\omega}_{*}\right)}\right)\right.} \\
& \left.\times\left(\frac{-i \hat{\nu}_{\eta} \hat{\omega}\left(\hat{\omega}+\tau \hat{\omega}_{*}\right)}{\left(\hat{\omega}-\hat{\omega}_{*}\right)}\right)^{1 / 2}-Z^{2}\right] \phi=0
\end{aligned}
$$

where

$$
Z=\left(\frac{\left(\hat{\omega}-\hat{\omega}_{*}\right)}{-i \hat{\nu}_{\eta} \hat{\omega}\left(\hat{\omega}+\tau \hat{\omega}_{*}\right)}\right)^{1 / 4} X .
$$

In the limit $D_{I} \gg \hat{\omega}\left(\hat{\omega}+\tau \hat{\omega}_{*}\right)$, the dispersion relation for the most unstable mode becomes

$$
\hat{\omega}\left(\hat{\omega}+\tau \hat{\omega}_{*}\right)\left(\hat{\omega}-\hat{\omega}_{*}\right)+i \hat{\nu}_{\eta} D_{I}^{2}=0 .
$$

In the absence of ion finite Larmor radius and electron diamagnetic effects, the normalized growth rate of the resistive interchange mode is

$$
\hat{\gamma}=D_{I}^{2 / 3} \hat{\nu}_{\eta}^{1 / 3} \propto D_{I}^{2 / 3} S^{-1 / 3} .
$$

On the other hand, in the presence of ion finite Larmor radius and electron diamagnetic effects with $\hat{\gamma}<\hat{\omega}_{*}$, the normalized growth rate of the resistive interchange mode is

$$
\hat{\gamma}=\frac{D_{I}^{2} \hat{\nu}_{\eta}}{\tau \hat{\omega}_{*}^{2}} \propto \frac{D_{I}}{S} .
$$

This result implies that in the presence of electron diamagnetic flow and finite Larmor radius effects, the resistive interchange mode grows on a slow time scale similar to that of equilibrium and that it is also independent of the wave number, similar to Refs. 7-9.

We have also solved Eqs. (22) and (23) numerically for different values of $D_{I}$ and $S$. Figure 4 shows that in the presence of resistivity, the interchange mode gets destabilized for $D_{I}>0$. Enhancement of the growth rate is observed with decreasing $S \propto 1 / \eta$ and this effect is more prominent at small $D_{I}$. For small $D_{I}$, the growth rate varies as $S^{-1 / 3}$, similar to the analytical result given by Eqs. (26) or (30) and is shown by the dashed lines in Fig. 4. For large $D_{I}$, the growth rate is approximately the same as that for the ideal interchange instability (shown by the solid line). In Fig. 5, we show that the electron diamagnetic and ion finite Larmor radius effects have a stabilizing effect on the resistive interchange mode for $S=10^{7}$ and $k_{y} a_{s}=0.02$. The normalized growth rate is significantly reduced for small $D_{I}\left(D_{I}\right.$ $\lesssim 0.4)$, while it has little or no effect for large $D_{I}(\gtrsim 0.5)$, especially for Lundquist number, $S \gtrsim 10^{6}$. 


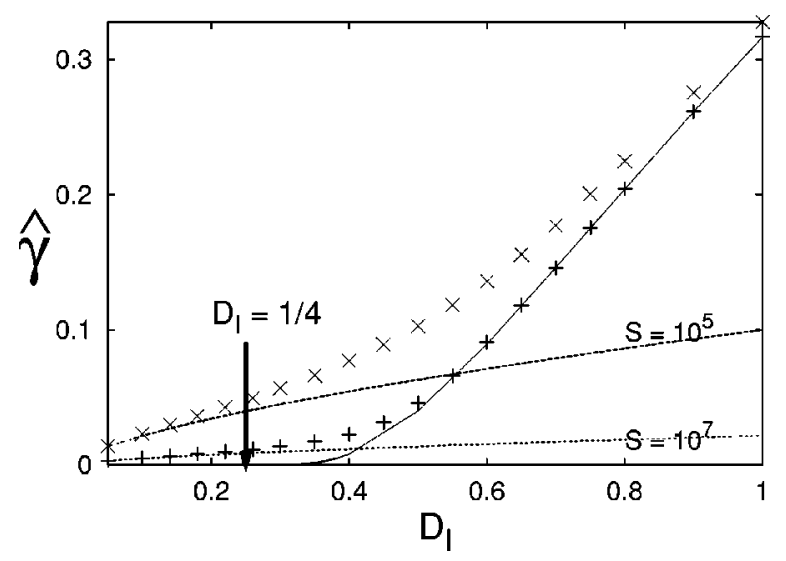

FIG. 4. In the presence of resistivity, the growth rate increases with decreasing Lundquist number $S$ as shown by + (for $S=10^{7}$ ) and $\times$ (for $S$ $=10^{5}$ ). Here, the solid line represents the growth rate for the ideal interchange mode and the dashed line represents the analytical result given by Eq. (30). For small $D_{I}(\lessgtr 0.4)$, the growth rate is similar to that of the resistive interchange instability (as shown by dashed lines); however, for large $D_{I}$ resistivity has a negligible effect on the ideal mode, particularly as $S$ increases.

\section{CONCLUSIONS}

In this paper, we have investigated the linear eigenmodes for localized ideal and resistive interchange modes in a simple sheared slab geometry. For the ideal interchange mode, a marginal stability boundary is observed at $D_{I}$ $=1 / 4$, which is in agreement with the Suydam criterion. ${ }^{1,2}$ However, this stability boundary is not a very restrictive condition due to an exponentially small growth rate for values of $D_{I}$ well above the marginal stability condition. Since the ideal MHD growth rate is small, the interchange mode can be easily stabilized by the finite ion Larmor radius effects. A connection between these results and the energy principle approach is made by using a quadratic form for the ideal interchange mode. This analysis indicates that for $D_{I}$ close to the marginal stability condition, the change in kinetic energy is very large because the mode is highly localized around

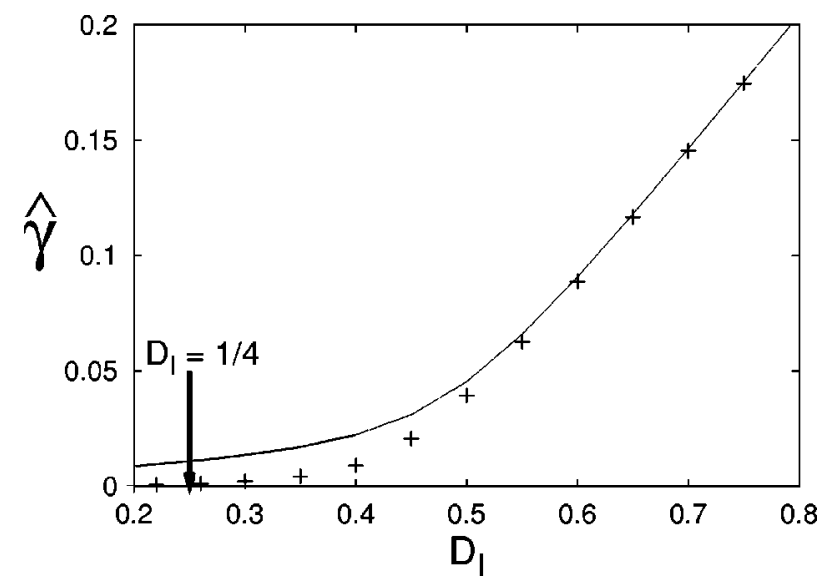

FIG. 5. Ion finite Larmor radius and electron diamagnetic flow effects have a stabilizing effect on the resistive interchange mode. The solid line represents the growth rate of the resistive interchange mode with $S=10^{7}$ and + shows the effect of electron diamagnetic and finite ion Larmor radius effects with $k_{y} a_{s}=0.02$.
$X=0$ (cf. Fig. 2), while the potential energy released from the pressure gradient is very small; hence the normalized growth rate $\hat{\gamma}_{\mathrm{MHD}}$ is negligibly small. For large $D_{I}$, the released potential energy varies quadratically with $D_{I}$, while the change in kinetic energy is nearly constant. This results in a linear variation of $\hat{\gamma}_{\mathrm{MHD}}$ with $D_{I}$ and is approximately given as $\hat{\gamma}_{\mathrm{MHD}} \simeq 0.6\left(D_{I}-0.46\right)$ for $D_{I}>0.5$. Due to the stabilizing effect of ion finite Larmor radius effects, the effective stability criterion for localized interchange instability with a robust MHD-like growth rate appears to be $D_{I}$ $\lessgtr 0.4-0.5$, not the Suydam criterion $D_{I}<1 / 4$.

In the presence of the resistivity, resistive interchange instability gets destabilized for $D_{I}>0$. In the weak resistivity case $\left(\hat{\omega} \sim \hat{\nu}_{\eta} / \Delta_{x}^{2}>\hat{\nu}_{\eta}\right)$, the normalized growth rate is $\hat{\gamma}$ $=\left[k_{y} \alpha\right]^{2 / 3} S^{-1 / 3}$ while in the resistivity-dominated case $(\hat{\omega}$ $\left.\ll \hat{v}_{\eta} \Delta_{\perp}^{2}\right)$, it is given by $\hat{\gamma}=\left(k_{y} D_{I}\right)^{2 / 3} S^{-1 / 3}$. The growth rate of these modes is significantly reduced in the presence of electron diamagnetic and ion finite Larmor radius (FLR) effects. Our analysis clearly shows that the nonideal effects such as resistivity, electron diamagnetic and ion FLR effects have a major effect only for small $D_{I}(\lesssim 0.4)$. In the presence of electron diamagnetic flow and ion finite Larmor radius (FLR) effects, the growth rate of the resistive interchange instability is proportional to resistivity $(\eta)$. It means that the mode grows very slowly, on the resistive equilibrium evolution time scale. For large $D_{I}(\gtrsim 0.5)$, the normalized growth rate is similar to that of an ideal interchange mode $\hat{\gamma}_{\mathrm{MHD}}$. Thus, we conclude that even in the presence of resistivity, the normalized growth rate of the interchange mode is negligible close to $D_{I}=1 / 4$ due to the stabilizing effect of electron diamagnetic flow and ion finite Larmor radius effects. Hence, with or without the nonideal effects such as resistivity, electron diamagnetic and ion finite Larmor radius effects, the effective criterion for robust ideal MHD instability ( $\hat{\gamma}_{\mathrm{MHD}}$ $>0.03)$ is $D_{I} \gtrsim 0.5$, a factor of about two greater than Suydam's instability criterion $\left(D_{I}>1 / 4\right)$.

Despite the long standing expectations that the Mercier criterion defines an operational limit for toroidal devices, there has been no definitive experimental evidence to support this. Indeed, indications from Stellarator experiments show that there is no observed degradation in the plasma properties when operated in regimes where $D_{I}>1 / 4 .{ }^{17}$ The calculations presented in this work offer a possible explanation. As suggested here, it would be of interest to compare experimental operation as $D_{I}$ transitions from marginal stability to higher values $\left(D_{I} \gtrsim 0.45\right)$, where more virulent instability is indicated.

\section{ACKNOWLEDGMENT}

This work was supported by the U.S. Department of Energy under Grant No. DE-FG02-86ER53218.

${ }^{1}$ J. P. Friedberg, Ideal Magnetohydrodynamics (Plenum, New York, 1987), Chaps. 9 and 10.

${ }^{2}$ B. R. Suydam, in Second United Nations International Conference on the Peaceful Uses of Atomic Energy (United Nations, Geneva, 1958), Vol. 81, p. 157.

${ }^{3}$ C. Mercier, Nucl. Fusion 1, 47 (1960).

${ }^{4}$ V. D. Shafranov and E. I. Yurchenko, Sov. Phys. JETP 26, 682 (1968).

${ }^{5}$ R. M. Kulsrud, Phys. Fluids 6, 904 (1963). 
${ }^{6}$ T. E. Stringer, Nucl. Fusion 15, 125 (1975).

${ }^{7}$ H. P. Furth, J. Killeen, and M. N. Rosenbluth, Phys. Fluids 6, 459 (1963).

${ }^{8}$ B. Coppi, Phys. Rev. Lett. 12, 417 (1964).

${ }^{9}$ B. Coppi, J. M. Greene, and J. L. Johnson, Nucl. Fusion 6, 101 (1966).

${ }^{10}$ M. N. Rosenbluth, N. A. Krall, and N. Rostoker, Nucl. Fusion Suppl. 1, 143 (1962).

${ }^{11}$ M. Abramowitz and I. A. Stegun, Handbook of Mathematical Functions (Dover, New York, 1964), Chap. 9.

${ }^{12}$ Y. B. Gott, M. S. Ioffe, and V. G. Telkovsky, Nucl. Fusion Suppl. 3, 1045 (1962).

${ }^{13}$ J. B. Taylor, Phys. Fluids 6, 1529 (1963).
${ }^{14}$ D. M. Meade, Phys. Rev. Lett. 17, 677 (1966).

${ }^{15}$ C. M. Bender and S. A. Orszag, Advanced Mathematical Methods for Scientists and Engineers (McGraw-Hill, New York, 1978), Chap. 9, p. 419.

${ }^{16}$ W. Magnus, F. Oberhettinger, and R. P. Soni, Formulas and Theorems for the Special Functions of Mathematical Physics (Springer-Verlag, Berlin, 1966), Chap. II.

${ }^{17}$ S. Okamura et al., in Proceedings of the 15th International Conference on Plasma Physics and Controlled Nuclear Fusion Research (International Atomic Energy Agency, Vienna, 1995), Vol. 1, p. 381. 